\title{
Functional evaluation of gene mutations in Long QT Syndrome: strength of evidence from in vitro assays for deciphering variants of uncertain significance
}

\author{
Jules C. Hancox ${ }^{1 *}$ D, Alan G. Stuart ${ }^{2,3}$ and Stephen C. Harmer ${ }^{1 *}$
}

\begin{abstract}
Background: Genetic screening is now commonplace for patients suspected of having inherited cardiac conditions. Variants of uncertain significance (VUS) in disease-associated genes pose problems for the diagnostician and reliable methods for evaluating VUS function are required. Although function is difficult to interrogate for some genes, heritable channelopathies have established mechanisms that should be amenable to well-validated evaluation techniques.

The cellular electrophysiology techniques of 'voltage-' and 'patch-' clamp have a long history of successful use and have been central to identifying both the roles of genes involved in different forms of congenital Long QT Syndrome (LQTS) and the mechanisms by which mutations lead to aberrant ion channel function underlying clinical phenotypes. This is particularly evident for KCNQ1, KCNH2 and SCN5A, mutations in which underlie > 90\% of genotyped LQTS cases (the LQT1-LQT3 subtypes). Recent studies utilizing high throughput (HT) planar patch-clamp recording have shown it to discriminate effectively between rare benign and pathological variants, studied through heterologous expression of recombinant channels. In combination with biochemical methods for evaluating channel trafficking and supported by biophysical modelling, patch clamp also provides detailed mechanistic insight into the functional consequences of identified mutations. Whilst potentially powerful, patient-specific stem-cell derived cardiomyocytes and genetically modified animal models are currently not well-suited to high throughput VUS study.

Conclusion: The widely adopted 2015 American College of Medical Genetics (ACMG) and Association for Molecular Pathology (AMP) guidelines for the interpretation of sequence variants include the PS3 criterion for consideration of evidence from well-established in vitro or in vivo assays. The wealth of information on underlying mechanisms of LQT1-LQT3 and recent HT patch clamp data support consideration of patch clamp data together (for LQT1 and LQT2) with information from biochemical trafficking assays as meeting the PS3 criterion of well established assays, able to provide 'strong' evidence for functional pathogenicity of identified VUS.
\end{abstract}

Keywords: Arrhythmia, Channelopathy, hERG, KCNH2, KCNQ1, Long QT syndrome, Variant of uncertain significance

\footnotetext{
* Correspondence: jules.hancox@bristol.ac.uk; s.charmer@bristol.ac.uk

'School of Physiology, Pharmacology and Neuroscience, Cardiovascular Research Laboratories, University of Bristol, Bristol, UK

Full list of author information is available at the end of the article
}

(c) The Author(s). 2020 Open Access This article is licensed under a Creative Commons Attribution 4.0 International License, which permits use, sharing, adaptation, distribution and reproduction in any medium or format, as long as you give appropriate credit to the original author(s) and the source, provide a link to the Creative Commons licence, and indicate if changes were made. The images or other third party material in this article are included in the article's Creative Commons licence, unless indicated otherwise in a credit line to the material. If material is not included in the article's Creative Commons licence and your intended use is not permitted by statutory regulation or exceeds the permitted use, you will need to obtain permission directly from the copyright holder. To view a copy of this licence, visit http://creativecommons.org/licenses/by/4.0/ The Creative Commons Public Domain Dedication waiver (http://creativecommons.org/publicdomain/zero/1.0/) applies to the data made available in this article, unless otherwise stated in a credit line to the data. 


\section{Introduction}

The now commonplace use of genetic screening for inherited cardiac conditions can be of high value in identifying the genetic substrate that underlies an observed clinical phenotype. However, such testing is not without problems: the term "genetic purgatory" has been used to describe the situation in which the genetic testrequesting physician, patients and their relatives are "left stuck" when a genetic variant is identified, the significance of which is unknown ('variant of uncertain significance', VUS) [1]. A VUS designation is made when there is insufficient information to distinguish between benign and pathological nucleotide changes [2]. Recognizing the increased complexity of genetic information consequent to the widespread adoption of next generation sequencing, the American College of Medical Genetics (ACMG) and Association for Molecular Pathology (AMP) published in 2015 standards and guidelines for the interpretation of sequence variants [3]. These guidelines contain a series of complementary criteria that can be used in combination to classify variants into one of five categories: 'pathogenic', 'likely pathogenic', 'uncertain significance', 'likely benign' and 'benign' [3]. According to this framework, variants designated as of uncertain significance are not to be used for clinical decision making [3]. These guidelines have also been adopted outside of the United States; for example, the UK Association for Clinical Genomic Science (ACGS) has adopted the 2015 ACMG/AMP guidelines and has itself provided best practice guidelines for variant classification that detail how ACMG/AMP evidence criteria should be addressed [4, 5].

Within the ACMG/AMP framework, the "PS3" criterion is used to consider results of "well-established in vitro or in vivo functional studies supportive of a damaging effect on the gene or gene product" [1]. With $\sim 20,000$ genes in the human genome, many genes will produce gene products for which (if present in the heart) the function is not well known, or for which there are not well-established functional tests. For proteins for which secondary/tertiary structures are available, either through high resolution microscopy or in silico homology modelling, it may be possible to predict likely changes to protein structure due to nucleotide coding changes. However, the ACMG/AMP and ACGS guidelines make clear that in silico studies, including protein modelling, cannot be used as functional evidence under the PS3 evidence criterion (they may instead be eligible for consideration under the phenotype specificity criterion PM1) [3, 4]. By contrast, the function of some gene products is very well established, through extensively validated, precise methods of investigating protein function. Ion channels are open to such interrogation. This article aims to consider recent evidence supporting the notion that cellular electrophysiological approaches can reliably distinguish between benign and pathological variants, and thus provide PS3 'strong' functional evidence for elucidating VUS in cardiac ion channelopathies.

\section{Channelopathies}

The term channelopathy encompasses a wide range of disorders that share in common a basis due to an acquired or genetic dysfunction in an ion channel. Channelopathies have been identified in the nervous system, cardiovascular system, skeletal muscle, urinary system and respiratory system [6]. Cardiac channelopathies include Long QT Syndrome (LQTS), Short QT Syndrome (SQTS), Brugada Syndrome (BrS), Catecholaminergic Polymorphic Ventricular Tachycardia (CPVT), familial atrial fibrillation (fAF) and some forms of sick sinus syndrome (SSS) [6, 7]. The two most widely studied channelopathies are probably cystic fibrosis (CF) and congenital forms of LQTS (cLQTS). Electrophysiological measurements of ion channel (dys) function have been central to the development of current understanding of both conditions. Although 17 forms of cLQTS have been identified, $\sim 95 \%$ of genotype positive cases involve mutations to 3 genes, $K C N Q 1, K C N H 2$ and $S C N 5 A$, which are considered to be definitively causally linked to LQT1-LQT3 ( [8, 9] and see Fig. 1). A reliable approach to functional interrogation of the products of these genes should therefore be of considerable value to deciphering VUS in cLQTS.

\section{Measurement of ion channel current- a brief historical perspective}

The quantitative measurement of ion channel currents through the voltage clamp technique is long-established, dating back well over 60 years [10-12] and for patch clamp over 40 years $[13,14]$. These techniques enable control of transmembrane voltage and direct measurement of transmembrane ion flux as electrical current, permitting accurate measurement of the amplitude, time-course and voltage dependence of current flow across cell membranes.

These methods were crucial for the early functional identification of the LQT1-3 subtypes of cLQTS. $\mathrm{KCNH} 2$ and KCNQ1 encode proteins that form the pore-forming subunits of the 'rapid' and 'slow' delayed rectifier potassium currents $\left(\mathrm{I}_{\mathrm{Kr}}\right.$ and $\left.\mathrm{I}_{\mathrm{Ks}}\right)$ that are important for normal ventricular repolarization ([15] and see Fig. 1). Native $\mathrm{I}_{\mathrm{Kr}}$ and $\mathrm{I}_{\mathrm{Ks}}$ were first pharmacologically resolved and characterized in ventricular cardiomyocytes using whole-cell patch clamp $[16,17]$. Whole-cell voltage clamp recording was subsequently used to identify KCNQ1 (alternative nomenclature KvLQT1, Kv7.1) as the pore-forming subunit of $\mathrm{I}_{\mathrm{Ks}}$ channels and the target of mutations causing the LQT1 variant of cLQTS $[18,19]$. It was also used to 


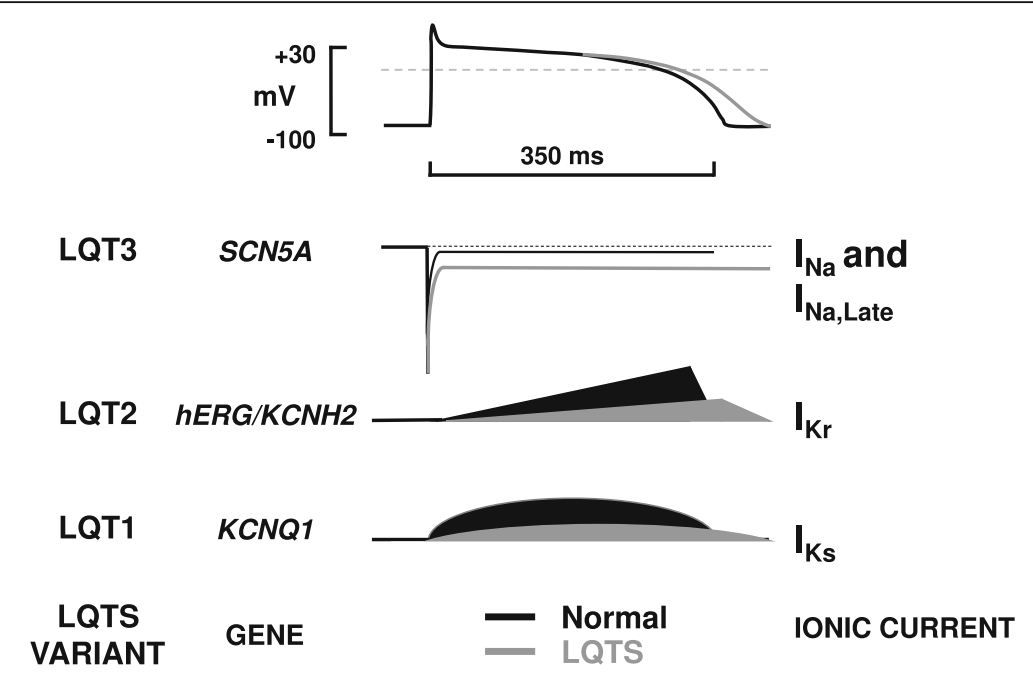

Fig. 1 Schematic diagram showing how ventricular repolarisation is prolonged and underlying ionic currents are altered by LQT1-LQT3 causing variants. Illustration of the effect of LQTS variants on the human ventricular action potential (AP) and the corresponding changes in the underlying currents. Upper panel: The ventricular AP is prolonged in congenital LQTS (types 1-3) (indicated by grey line) due to either an inappropriate gain of function (GOF) in late sodium current ( $\left.I_{\mathrm{Na}, L \text { ate }}\right)$ or a loss of function (LOF) in the slowly $\left(\mathrm{I}_{\mathrm{KS}}\right)$ or rapidly $\left(\mathrm{I}_{\mathrm{K}}\right)$ activating delayed rectifier potassium currents. Lower panel: Effect of LQTS causing variants in SCN5A, KCNH2 (hERG) or KCNQ1 on the magnitude and temporal profile of the sodium current $\left(I_{\mathrm{Na}}\right)$ or potassium currents $\left(I_{\mathrm{Kr}}\right.$ or $\left.I_{\mathrm{Ks}}\right)$ respectively. The normal current profiles are shown in black and the currents produced in the presence of LQTS causing variants are displayed in grey. The relative contributions of each current are not drawn to scale

determine that the $\mathrm{KCNH} 2$-encoded hERG protein (alternative nomenclature Kv11.1) underlies the pore of the $\mathrm{I}_{\mathrm{Kr}}$ channel and is the target for LQT2 mutations [20-23]. Voltage clamp of SCN5A encoded Nav1.5 channels was important for the demonstration of the role of SCN5A gain-of-function mutations in LQT3 [24-26] and, later, of the role of $S C N 5 A$ in $\mathrm{BrS}$ [27]. It is notable that approaches to the interrogation of the propensity for novel drugs to produce an acquired LQTS and Torsades de Pointes arrhythmia also involve patch clamp assays [28-30].

Manual patch clamp has been used in much of the academic/preclinical research that underpins current understanding of LQTS and other channelopathies, though a detailed coverage of the numerous studies involving manual patch clamp is outside the intended scope of the present article. However, taking KCNH2-mediated LQT2 as an illustrative example, a combination of hERG protein measurement (using Western blotting) and manual patch clamp was used to demonstrate that the majority (28/34) of misense mutations exhibit a class 2 (impairment of the intracellular processing and trafficking of channels to the cell membrane [31]) phenotype and demonstrate the potential for pharmacological correction. It also showed that a small number of missense mutations do not exhibit a loss-of-function phenotype, thus highlighting the necessity for functional studies to interpret the effects of individual variants [32]. A subsequent large scale analysis of 167 LQT2-linked hERG mutations used biochemical analysis supported by manual patch clamp of selected variants to demonstrate that a class 2 mechanism mediates the effects of most (close to $90 \%$ ) mutations in 3 of 4 major structural domains of the hERG channel (the exception being the distal C-terminus) [33]. At the time it was conducted this study was the largest mutational study for any inherited disease [33].

\section{Deciphering VUS using automated patch clamp}

Despite the pivotal value of cellular electrophysiology in understanding ion channel function, such methods are not routinely used to study genotyped variants identified in channelopathies. This is because, as highlighted recently by others [2], the gold standard approach of manual patch clamp is both labour- and time-intensive. A potential solution is to employ automated, planar patch clamp technology. Automated patch clamp approaches have been largely driven by the need to screen large numbers of compounds for ion channel effects during drug development [28]. Automated patch clamp has its own technical challenges including incorporation of temperature control and obtaining and maintaining high electrical resistance seals necessary to secure reliable voltage clamp; however, in principle, it has the potential to offer a means of interrogating channel variants in volume, significantly faster than is possible with conventional, manual patch clamp. This is supported by recent work with KCNQ1, KCNH2, and SCN5A (summarised in Table 1).

There are over 600 disease-associated $K C N Q 1$ variants [37], but only a small proportion of these have been 
Table 1 Automated patch clamp technology enables the phenotyping of KCNQ1, KCNH2 and SCN5A variants to be performed in a high-throughput manner

\begin{tabular}{|c|c|c|c|c|c|c|c|c|}
\hline \multirow[t]{2}{*}{ Study: } & \multirow{2}{*}{$\begin{array}{l}\text { Expression } \\
\text { system: }\end{array}$} & \multirow[t]{2}{*}{ Automated patch clamp platform: } & \multicolumn{2}{|c|}{ No of variants studied: } & \multicolumn{4}{|c|}{ Classification: } \\
\hline & & & Homozygous state: & $\begin{array}{l}\text { Heterozygous } \\
\text { State: }\end{array}$ & P or LP: & B or LB: & GOF: & VUS: \\
\hline KCNQ1 [34] & $\mathrm{CHO}-\mathrm{K} 1$ & Syncropatch 768 PE - Recording at RT & $\begin{array}{l}78 \text { (30 training }+48 \\
\text { test variants) }\end{array}$ & 22 & 44 & 16 & 3 & 15 \\
\hline KCNH2 [35] & HEK-293 & Syncropatch $384 \mathrm{PE}$ - Recording at $\sim 25^{\circ} \mathrm{C}$ & 23 & 30 & $26^{\mathrm{a}}$ & $2^{\mathrm{a}}$ & $0^{\mathrm{a}}$ & $2^{\mathrm{a}}$ \\
\hline SCN5A [36] & HEK-293 T & Syncropatch 384 PE - Recording at RT & $\begin{array}{l}83 \text { ( } 73 \text { previously } \\
\text { unstudied) }\end{array}$ & N/A & 54 & 17 & 0 & 12 \\
\hline
\end{tabular}

P Pathogenic, LP Likely Pathogenic, B Benign, LB Likely Benign, GOF Gain of Function, VUS Variant of Uncertain Significance. KCNQ1 variant classification is inferred from in text information and data presented in Supplementary Tables S4a and S4b [34]. KCNH2 variant classification is as reported in [35]. ${ }^{a}$ Indicates that pathogenicity was classified in the heterozygotic state to mimic the patient phenotype [35]. SCN5A variant classification is taken from Supplementary Table S1 [36]. The Syncropatch 384 and 768 PE automated patch clamp platforms are developed by Nanion Technologies

functionally characterized. In order to evaluate the utility of automated patch clamp for elucidating the pathogenicity of VUS, Vanoye et al studied 78 known KCNQ1 variants, including 30 training and 48 test variants [34] (Table 1). Automated and manual patch clamp recordings were made at room temperature from mammalian (CHO-K1) cells transfected with wild-type (WT) or mutant KCNQ1 (together with its known $\beta$ subunit, KCNE1). Where comparative data on mutant effects on current amplitudes and kinetics were available for both manual and automated methods, there was generally a strong concordance between the two [34]. As most cases of LQTS are heterozygous (autosomal dominant or de novo), and functional $I_{\mathrm{Ks}}$ channels are tetrameric, the authors also studied 29 variants as WT/variant heteromultimers through WT:mutant co-transfection (7 variants could not be analysed due to confounding expression effects, leaving 22 for analysis). Of these, 17 showed current densities similar to those carried by the mutant expressed alone, indicative of dominant negative pathogenicity. Interestingly, two variants failed to show significant differences from WT current when studied in co-expression experiments (as also seen reported for some KCNQ1 variants in [38]), highlighting the importance of performing experiments to mimic heterozygous conditions [34]. This approach enabled approximately two-thirds of the variants studied to be reclassified based on functional evidence (Table 1).

There are over $1000 \mathrm{KCNH} 2$ variants in public databases and, similar to the situation for KCNQ1, only a relatively small fraction of the known variants has been subject to functional evaluation. There appear to be differences in severity of the effects of mutations in different regions of the hERG channel, with mutations in the pore region associated overall with higher risk than mutations in non-pore regions $[39,40]$. A recent study has employed automated patch clamp recording to determine whether this approach may distinguish pathological from benign non-synonymous variants [35] (Table 1) and twinned this with a high-throughput channel trafficking assay for comparison. hERG channels were expressed in mammalian (HEK-293) cells and through use of a bicistronic expression vector, wild-type and mutant channels could be co-expressed to mimic heterozygous heritability. Of the 30 variants studied in the heterozygotic state, 20 had designations of pathogenicity or otherwise in ClinVar on the basis of segregation/ bioinformatic predictions (16 as pathogenic, 1 benign and 3 VUS). For these 20 variants the predictions of the patch clamp experiments demonstrated very high concordance with the ClinVar classifications [35]. For the ten variants that had not received classification in ClinVar, eight of these showed changes consistent with clear pathogenicity and two displayed defects that were less marked but consistent with likely pathogenic [35]. Overall, the results of this study highlight the value of patch clamp in distinguishing pathogenic changes to hERG channel function.

A 2018 comprehensive literature review showed a clear correlation between electrophysiological parameters of SCN5A-encoded Nav1.5 channel current and BrS and LQT3 [41]. Three hundred fifty-six variants were found for which electrophysiological parameters were available: peak current, late current, voltage dependent activation and inactivation and recovery from inactivation. The authors also quantified carriers presenting with/without disease. Peak current and late current respectively correlated with $\mathrm{BrS}$ and LQT3 penetrance [41]. Half-maximal activation voltage $\left(\mathrm{V}_{0.5}\right)$ and recovery from inactivation respectively associated with $\mathrm{BrS}$ and LQT3 penetrance [41]. More recently, automated patch clamp has been applied to 83 SCN5A variants: 10 control variants that had been studied previously; 10 suspected benign variants and 63 suspected $\mathrm{BrS}$ associated variants [36]. Variants showing $<50 \%$ peak current density of WT or with a $>+10 \mathrm{mV}$ shift in voltage-dependent activation were considered to have significant loss of function (and thereby to meet the ACMG/AMP PS3 criterion) [36]. Twenty-two of the sixty-three suspected $\mathrm{BrS}$ loss of function mutations had $<10 \%$ peak current; 
another 23 had partial loss of function $(10-50 \%$ peak current). Of the 73 previously unstudied variants, 60 were classified in Clin Var as VUS (with 11 likely pathogenic and 2 likely benign) prior to patch clamp analysis. After patch clamp had been performed, 47 were classified as pathogenic/likely pathogenic, 16 were benign/ likely benign and 10 were VUS [36]. Additional use of structural modelling suggested potential mechanisms for loss of function with particular mutations [36]. Overall, as for KCNQ1 and KCNH2, SCN5A VUS appear amenable to elucidation through high throughput patch clamp approaches.

The above studies highlight the feasibility and comparative speed of using automated patch clamp technology to predict variant pathogenicity in a highthroughput manner. For example, in their study Vanoye and colleagues estimated that $12 \mathrm{KCNQ} 1$ variants could be generated and evaluated within the very rapid timeframe of 2 weeks [34], though presumably this estimate involves some further streamlining, as the study of 48 homomeric variants occupied $\sim 12$ weeks. A key contribution of these studies [34-36] is to demonstrate the feasibility of functional electrophysiology data-driven variant classification and the associated ability to 'decrypt' VUS. Indeed, with reference to the ACMG/AMP guidelines, the authors of the Vanoye et al study commented "Among the criteria weighted as strongly supportive of classifying variants as pathogenic is the availability of data from in vitro or in vivo functional studies demonstrating a damaging effect on the gene or gene product (PS3 criterion)", continuing "Our approach to high throughput functional evaluation of KCNQ1 variants offers a rich new data source that satisfies the PS3 criteria and can aid variant classification" [34].

\section{Heterologous cell-based patch-clamp analysis- still a gold standard?}

It should be clear from the foregoing text that patch clamp investigation of recombinant channels is likely the most direct approach to provide insight into functional consequences of mutations to KCNQ1, KCNH2 and $S C N 5 A$ and, by extension, to other genes (e.g. RYR2 [42]) that encode pore forming subunits of cardiac ion channels. The functional outcomes are particularly enhanced when patch-clamp is combined with complementary biochemical approaches that can interrogate trafficking status. However, heterologous expression removes the channel from the normal cardiomyocyte environment that may contain other channel accessory proteins and signalling pathways or express genetic modifiers of disease severity (e.g. [43]) that are absent in a heterologous expression system. An important question that therefore arises is whether or not there are superior methods for investigating channel function to inform rare variant classification?

A potential approach to address the cellular context of variants is to use a genetically modified animal model in which the target mutation can be engineered, either by modification of the orthologous gene in that species, or through transgenic expression of the human gene. Unfortunately, where $K C N H 2$ and $K C N Q 1$ are concerned, this is not straightforward. The most commonly used mammalian species for genetic modification is the mouse and mice possess abbreviated ventricular action potentials that differ markedly in profile from those in humans and that do not rely on $\mathrm{I}_{\mathrm{Kr}}$ or $\mathrm{I}_{\mathrm{Ks}}$ for repolarization [44]. For this reason, mice are precluded from use in cardiac safety testing of new drugs [45] and they are similarly unsuitable for studying $K C N H 2$ and KCNQ1 mutations. Transgenic rabbit models of LQT1 and LQT2, as well as $\mathrm{KCNH}$ 2-linked short QT syndrome have been made and used to interrogate mechanisms of arrhythmia in these syndromes (e.g. [46-49]). However, the manufacture of such models is expensive and timeconsuming and, at present, impractical for routine functional investigation of rare variants. Zebrafish possess an orthologue of hERG and the $z E R G$ break-dance trafficking mutant exhibits a prolonged QT interval [50-52]. Also, knockdown of endogenous zERG channels in embryonic and larval zebrafish and expression of wild-type and variant hERG has been suggested to provide a method for in vivo study of $\mathrm{KCNH} 2$ mutations and polymorphisms [53, 54]. KCNQ1 mediated channels are expressed in zebrafish heart, but the $\mathrm{I}_{\mathrm{Ks}}$ channel $\beta$ subunit, KCNE1 is expressed at only low levels $[55,56]$. A transgenic SCN5A zebrafish model manifested bradycardia and conduction system abnormalities with a mutant but not wild-type human SCN5A construct [57]. Zebrafish therefore have potential for interrogating LQT1LQT3 mutations and the production of zebrafish models is likely to be much less expensive than of transgenic rabbits. The ability to undertake detailed interrogation of mutation consequences in adult zebrafish hearts is likely to be mutation-dependent, however, and not to be high throughput.

A promising alternative is the use of patient derived induced pluripotent stem-cell (iPSC) derived cardiomyocytes (hiPSC-CMs) and/or to use genetic modification approaches (CRISPR-Cas9) to introduce a mutation against an otherwise isogenic iPSC background (e.g. [58-68]). However, this approach still involves considerable lead-in time to produce viable myocytes, which show an immature phenotype, including a lack or paucity of the inwardly rectifying potassium current, $\mathrm{I}_{\mathrm{K} 1}$, that sets the resting potential in adult cardiomyocytes and spontaneous activity (and corresponding immature action potential morphology) that is absent in adult 
cardiomyocytes [68-70]. The variable morphology and duration of action potentials produced by individual hiPSC-CMs [71] (due to developmental immaturity and 'sub-type specification') also make it challenging to determine whether observed differences are attributable to effects of the variant being studied or to sampling considerations. Additionally, the comparatively small size of endogenous $\mathrm{I}_{\mathrm{Kr}}$ and $\mathrm{I}_{\mathrm{Ks}}$ and need to pharmacologically isolate them from overlapping currents makes precise characterization of kinetic changes more difficult than in heterologous systems. Thus, whilst genetically-modified animals and hiPSC-CMs may have utility for studying disease mechanism and potential patient-specific treatment approaches, they cannot yet supplant the study of heterologously expressed channels.

\section{Biophysical and computational modelling}

It is worth here highlighting the additional utility of biophysical modelling [72] to contextualize results obtained with recombinant channel electrophysiology. Correctly used, biophysical modelling is qualitatively distinct from computational modelling of protein structure or the use of bioinformatic tools that aim to predict pathogenicity, neither of which can provide direct functional insights. A number of human ventricular action potential models exist that are based on experimental data (e.g. [73-76]). Modification of the formulations that describe specific ionic currents, based on experimental data, can accurately reproduce changes to current density and kinetics observed in recombinant mutant channel recordings. The consequences of these changes for overall electrophysiological behaviour can then be interrogated. Computational cardiology approaches range from single cell to organ level simulations [72]. A recent example of the utility of combining electrophysiological, biochemical and modelling approaches comes from a study of KCNH2 variants identified from genetic testing of 292 cases of sudden infant death syndrome (SIDS). Voltage clamp and Western blot data on 4 identified variants showed similar functional expression levels as WT channels, but accelerated activation and deactivation gating in two mutants. Incorporation of these changes into a ventricular action potential model showed that the modest kinetic changes observed did not result in significant action potential changes, suggesting that the mutations found may not have been causally linked to SIDS [77]. Thus, whilst biophysical modelling cannot reasonably be used in isolation for functional evaluation of variant effects, when it is used to evaluate consequences of altered currents established in heterologous expression experiments, it is a valuable component of the overall functional evaluation.

In the absence of biophysical modelling, conventional voltage clamp can usefully be combined with the action potential voltage clamp technique ("AP clamp"), in which conventional rectangular waveforms are replaced with cardiac action potential waveforms as voltage commands (e.g. [78-80]). This technique takes into account membrane potential "history" [81] and thus elicits currents with their normal physiological time-course and voltage-dependence. AP clamp can therefore be used to obtain a "snapshot" of how a mutation influences the contribution of a current during repolarisation. By way of illustration, Fig. 2a and b show examples of $\mathrm{I}_{\mathrm{hERG}}$ carried by WT hERG and WT hERG co-expressed with a recently reported loss-of-function variant (T634S), showing reduced current under conventional voltage clamp (Fig. 2a) and under AP clamp (Fig. 2b). In this example, the further incorporation of the observed extent of current into a human ventricular AP model was used to demonstrate the repolarisation delay consequent to $\mathrm{I}_{\mathrm{hERG}} / \mathrm{I}_{\mathrm{Kr}}$ reduction (Fig. 2c). A file with example AP waveforms that can be downloaded and imported into voltage clamp acquisition software to conduct AP clamp experiments is freely available as an online supplementary appendix to [83].

\section{Validated approaches to meet the ACMG/PS3 "strong" criteria?}

The inherent challenge in interpreting what represents "well-established in vitro or in vivo functional studies supportive of a damaging effect on the gene or gene product" [1] to match the PS3 'strong evidence' code (and its benign counterpart, BS3) has recently led the Clinical Genome Resource (ClinGen) Sequence Variant Interpretation (SVI) Working Group to propose recommendations for evaluators of the clinical validity of functional data [84]. The steps include: (i) define disease mechanism; (ii) evaluate the applicability of general classes of assays used in the field; (iii) evaluate the validity of specific instances of assays and (iv) apply evidence to individual variant interpretation [84]. In broad terms these steps seem reasonable and in the case of LQT1-3, at least, it should be clear that the nature of the channel subunit dysfunction is amenable to elucidation through both manual and automated patch clamp. However, the recommendations also consider benchmarking through inclusion of known pathogenic and benign variants, suggesting inclusion of a total of 11 pathological and benign variants to arrive at a PS3 moderate evaluation [84]. The validity of patch clamp for the study of $K C N H 2, K C N Q 1$ and SCN5A mutations has already been demonstrated on well over this number of variants for LQT1-3 and, in principle, the inclusion of 11 control variants in automated patch clamp would not represent an excessive additional workload. That said, relatively few centres outside the pharmaceutical industry possess automated patch clamp platforms and for manual patch clamp 
A Conventional voltage clamp:
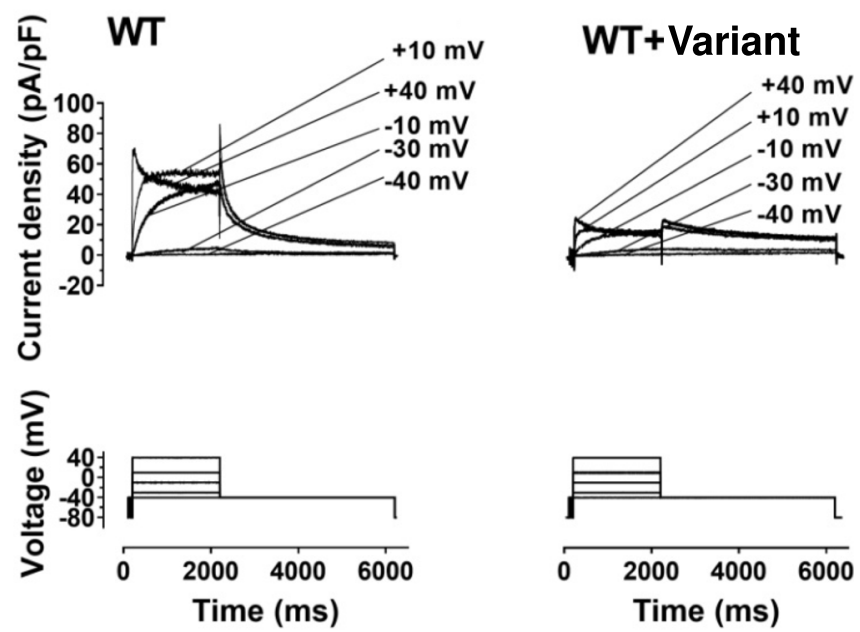

B Action potential voltage clamp:

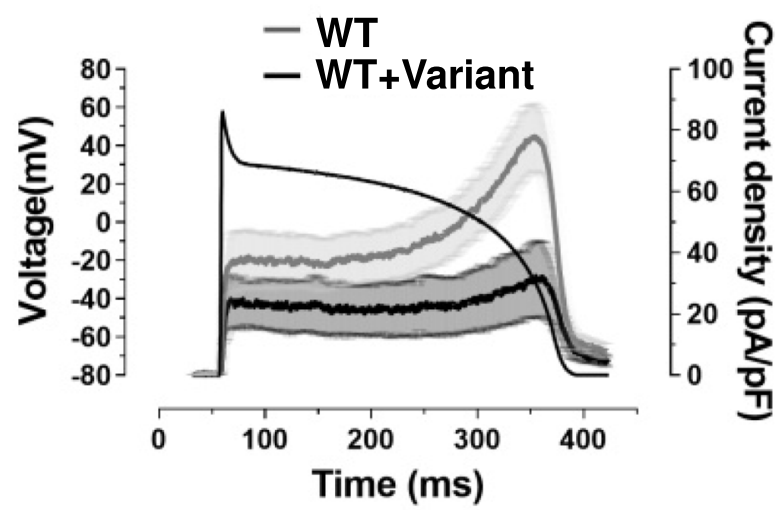

C Biophysical (action potential) modelling:

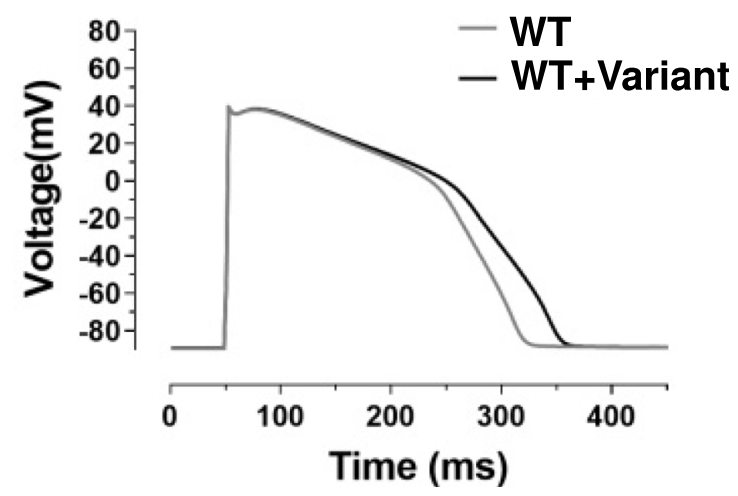

Fig. 2 (See legend on next page.) 
(See figure on previous page.)

Fig. 2 Conventional voltage and action potential (AP) clamp combined with biophysical AP modelling. a Shows families of hERG currents (I $\mathrm{h}_{\mathrm{h} R \mathrm{R} i}$ upper panels) elicited by a series of depolarising voltage commands to a range of test potentials (lower panels) under conventional voltage clamp. Numbers next to ionic current records denote voltage command potential. Left hand side shows WT I hERG data, whilst right hand side shows data for co-expression of WT channel with a loss-of-function variant (T634S [82]). Currents under the two conditions are shown on the same scale, indicating a marked loss-of-function effect of the missense mutation studied. $\mathbf{b}$ Shows profile of WT I $_{\text {hERG }}$ compared with that of

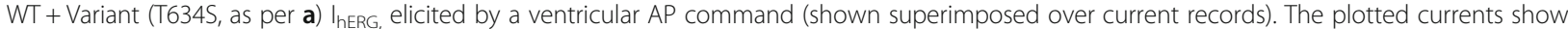
mean \pm SEM data from 5 experiments under each condition. The method allows direct visualization of the effect of this variant - producing a reduction in current throughout the AP plateau and repolarisation phase. c Shows the consequences of incorporation of the T634S hERG variant effect on ventricular AP repolarisation, using a human ventricular AP model. $I_{\mathrm{Kr}}$ was scaled to reflect the extent of current reduction found in voltage clamp experiments to mimic heterozygous variant expression conditions. This led to delayed repolarisation and AP prolongation. For further details see [82]

characterization of a new variant, the inclusion of an additional 11 control variants would represent both an unrealistic and entirely unnecessary burden. Our view is that the experimental approach is sufficiently well validated for the pathologies in question that this is not required. A more realistic scenario for a novel channel variant is comparison with the WT channel under homozygous expression conditions and under WTvariant (heterozygous) co-expression conditions, with inclusion of a well-established pathological mutation as a comparator. This is consistent with independent comments on the use of heterologous expression for variant classification that "Mammalian transient transfection studies are widely used as functional studies for missense variants and are generally considered strong evidence provided that the experiments include appropriate controls such as a positive wild-type control, a negative vector alone, a correction for transfection efficiency with multiple replicate experiments, and ideally, known pathogenic and benign variants. When evidence suggests a dominant negative mechanism, the experimental set up should include a 50:50 heterozygous mix to explore multimer disruption" [85].

The working group's recent guidance on 'stacking evidence' is conservative: where multiple functional assay results are available for a single variant, the evaluator is advised to apply evidence from the assay that is most well validated [84]. The committee was unable to reach consensus on whether results from different types of functional assay could be combined [84]. In the case of an ion channel gene mutation that leads to a trafficking defect, biochemical assays can show whether or not cell surface expression of the protein is reduced (and also whether the total amount of the protein in the cell is changed or not), whilst patch clamp shows the extent to which net ionic current through the channels in the membrane is reduced. These are complementary measures that are (a) mutually supportive and (b) combine to provide mechanistic insight into the nature of channel dysfunction present. It would thus be erroneous to evaluate the combination of evidence from the combination of the two approaches as equivalent to the strength of one of them alone. Furthermore, once functional consequences have been further explored through biophysical modelling, a clear result from the combined approach should exceed any reasonable threshold for 'strong' evidence. Indeed, in recent work on a $\mathrm{KCNH} 2$ variant found on molecular autopsy, the combination of manual patch clamp characterization and surface expression without modelling was considered to meet the PS3 'strong' criterion [86]. Fig. 3 summarizes an integrated approach to the functional interrogation of suspected LQT1-3 variants, utilizing recombinant channel approaches. This scheme does not preclude additional work with hiPSC-CMs and/or genetically modified animals, where these are available; rather it focuses on utilizing recombinant channel data to best effect.

\section{Conclusions}

Vanoye and George have recently highlighted that whilst reclassification of ion channel variants using data from functional assays conforms with the ACMG guidelines, and classification of a $\mathrm{KCNH} 2$ variant on the basis of dominant negative loss of function behaviour is reasonable, some caveats are necessary [2]. In particular, a failure to find a functional or biochemical change in a heterologous expression assay does not necessarily entirely exclude pathogenicity [2]. For example, dysfunction might only become evident under particular stressors such as adrenergic drive [2], or in the presence of additional genetic modifiers [87]. Moreover, functional evidence is only one part of the overall picture and however strong it may be, it does not directly address issues of penetrance or expressivity. We have focused on KCNQ1, KCNH2 and SCN5A as the function of these genes is fairly well-understood. The issue of what constitutes definitive evidence may be more difficult for other LQTS subtypes where the causal links between gene mutation and disease phenotype are less well-established [9]. Functional studies also have to be considered with available information on penetrance/expressivity. For example, in a recent study of KCNE1linked LQT5, ECG penetrance of heterozygotic family members was only $\sim 20 \%$ [88], so many mutation 


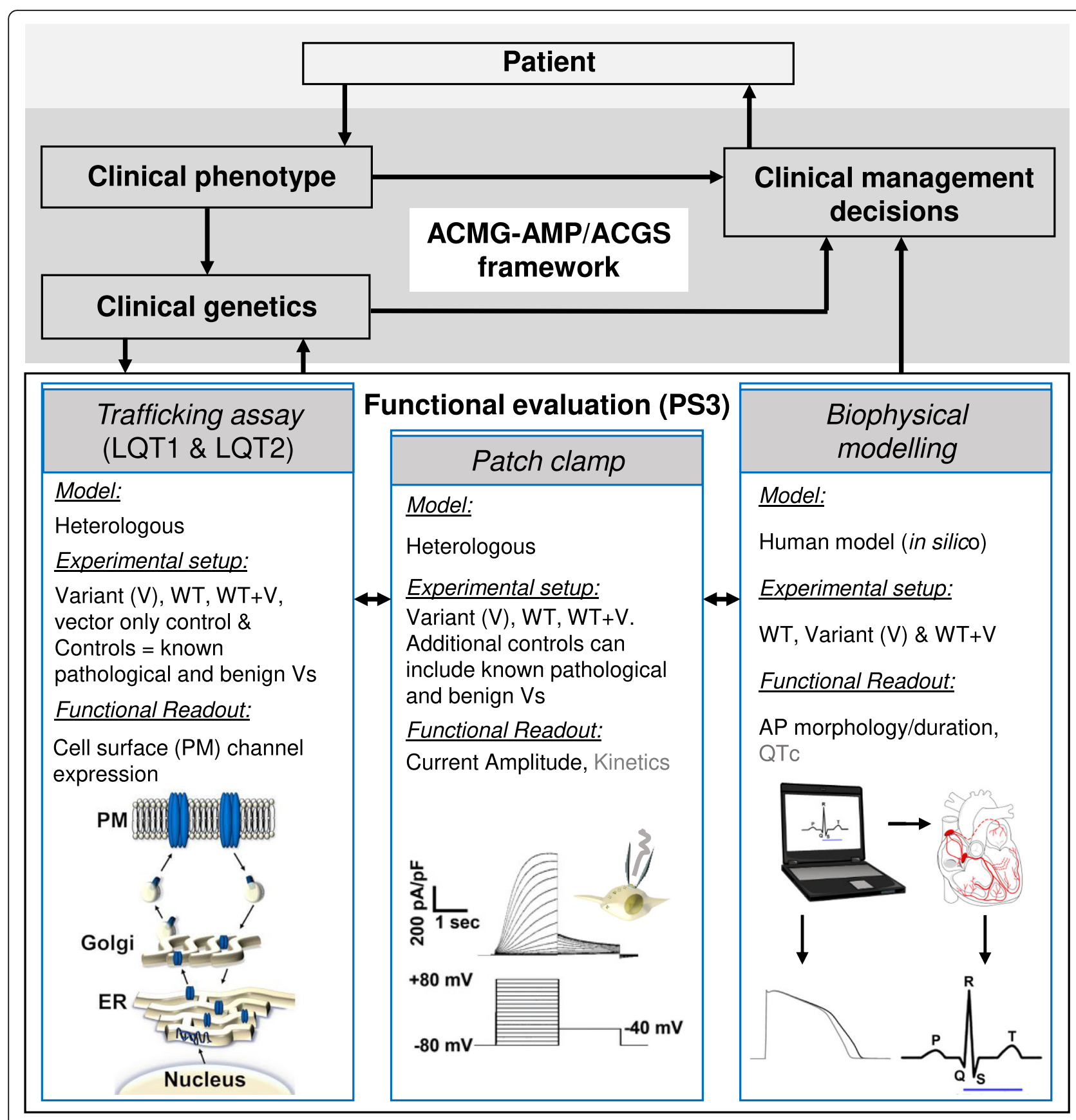

Fig. 3 (See legend on next page.) 
(See figure on previous page.)

Fig. 3 Schematic diagram of integrated approach to VUS evaluation, incorporating functional evaluation of recombinant channels. Clinical evaluation, establishing a clear clinical phenotype is the key driver of clinical management. Where the clinical phenotype suggests LQTS, interrogation of known LQTS genes proceeds through clinical genetics. The flow chart assumes that results of such analysis yields a VUS in KCNQ1, KCNH2, or SCN5A. For all 3 genes, previously uncharacterized variants can be investigated using whole-cell patch clamp measurements of current carried by recombinant channels heterologously expressed in a mammalian expression system. At the minimum, experiments should involve multiple replicate recordings from WT and variant $(V)$ channels separately and with equal co-expression of $W T+V$ to mimic the heterozygous state. Key measurements are of current size (which can be normalized to cell surface area, as cell capacitance, to facilitate pooling of data from the different experiments) and time-course. Differing conventional square-wave voltage clamp protocols can be used to enable quantification of amplitude and kinetics. Action potential voltage clamp will give a direct 'snapshot' of effect of the variant on current profile during a physiological waveform. For KCNQ1 and $\mathrm{KCNH} 2$ variants, a complementary trafficking assay should be performed allowing quantification of expressed mature/surface channel protein. Such a trafficking assay should use the same range of WT and variant expression conditions as patch clamp, ideally also including vector only control and known LQTS variants as controls. Biophysical modelling may not be required where variants produce a large dominant negative effect on channel expression, but it is useful in all cases and necessary where the predominant effects under patch clamp are on current kinetics. At the minimum, such modelling should investigate variant effects on AP duration in a human ventricle model. More sophisticated multicellular modelling can investigate effects on simulated QT interval. Modelling should include conditions to mimic heterozygous inheritance. The integrated results from functional evaluation can inform overall variant classification of pathogenicity and inform clinical management

carriers may be unaffected. This underscores the key role for clinical phenotype in diagnosis [1]. Additionally, whilst KCNE1 is well-accepted to be the $\beta$-subunit of the $I_{K s}$ channel and LQT5 mutations are likely to manifest in studies of KCNQ1 and KCNE1 [89, 90], KCNE1 can also co-assemble with the hERG channel and KCNE1 mutations may rarely adversely affect $\mathrm{I}_{\mathrm{Kr}}$ function [91-94]. The situation is potentially more complex for KCNE2, as this can produce promiscuous interactions with multiple different ion channels $[95,96]$ and require co-expression with multiple different ion channel partners to identify/exclude a pathogenic functional effect.

Therefore, both the design and interpretation of strength of evidence from in vitro functional experiments must consider fully what is known about the role of the protein in question. Ultimately, it may be difficult to arrive at a single adapted PS3 criterion that applies equally to all heritable arrhythmias, or indeed to all genes associated with a single arrhythmia syndrome. Disease-specific adaptation of the ACMG/AMP guidelines has been undertaken for $M Y H 7$-associated cardiomyopathies, including adaptation of the PS3 criterion [97]. Such adaptation is likely to be useful for heritable LQTS and other inherited arrhythmia syndromes. Incorporation of specialist knowledge of the available, established functional/biochemical assays is required to optimise disease-specific guidance for the PS3 criterion application. This would ensure that the strength of functional/biochemical evidence is assessed using best-inpractice, realistic methods and enable accurate dissemination of the weight of supporting evidence to direct variant classification and therefore the overall decisionmaking by clinicians. The price of failing to correctly identify a VUS as benign is the incorrect attribution of causality, whilst that of failing to classify a pathological
VUS as 'pathological' or 'likely pathological' is the omission of information that may have utility for clinical management.

\section{Acknowledgements}

Figure 2 is reproduced and modified from [82] (doi: https://doi.org/10.1016/j. bbrc.2020.04.003) under a Creative Commons Attribution 4.0 (CC BY 4.0) license.

\section{Authors' contributions}

JCH and SCH conceived and drafted the article. AGS helped draft and revise the article critically for important intellectual content. All authors approved the final version of the article.

\section{Funding}

No specific funding was involved in the preparation of this article. The authors acknowledge the British Heart Foundation for research laboratory funding.

\section{Availability of data and materials}

Review article: N/A.

Ethics approval and consent to participate

Review article: N/A.

\section{Consent for publication}

Review article: N/A.

\section{Competing interests}

AGS is Editor-in-Chief of the Journal of Congenital Cardiology.

\section{Author details}

${ }^{1}$ School of Physiology, Pharmacology and Neuroscience, Cardiovascular Research Laboratories, University of Bristol, Bristol, UK. ${ }^{2}$ Bristol Royal Hospital for Children, Bristol, UK. ${ }^{3}$ Bristol Heart Institute, University Hospital Bristol, Bristol, UK.

Received: 2 April 2020 Accepted: 13 August 2020

Published online: 05 November 2020

References

1. Ackerman MJ. Genetic purgatory and the cardiac channelopathies: exposing the variants of uncertain/unknown significance issue. Heart Rhythm. 2015; 12(11):2325-31. 
2. Vanoye $\mathrm{CG}$, George $\mathrm{AL} J \mathrm{~J}$. Decoding $\mathrm{KCNH} 2$ variants of unknown significance. Heart Rhythm. 2019;17(3):501-2.

3. Richards S, Aziz N, Bale S, Bick D, Das S, Gastier-Foster J, et al. Standards and guidelines for the interpretation of sequence variants: a joint consensus recommendation of the American College of Medical Genetics and Genomics and the Association for Molecular Pathology. Genet Med. 2015; 17(5):405-24.

4. Ellard S, Baple EL, Berry E, Forrester N, Turnbull C, Owens M, et al. ACGS Best Practice Guidelines for Variant Classification 2019. Assoc Clin Genomic Sci. 2019:1-32. https://www.acgs.uk.com/quality/best-practice-guidelines/.

5. Ellard S, Baple EL, Callaway A, Berry I, Forrester N, Turnbull C, et al. ACGS Best Practice Guidelines for Variant Classification in Rare Disease 2020. Assoc Clin Genomic Sci. 2020:1-33. https:/www.acgs.uk.com/quality/best-practiceguidelines/.

6. Kim JB. Channelopathies. Korean J Pediatr. 2014;57(1):1-18.

7. Campuzano O, Brugada R. Genetics of familial atrial fibrillation. Europace. 2009;11(10):1267-71.

8. Barsheshet A, Dotsenko O, Goldenberg I. Congenital long QT syndromes: prevalence, pathophysiology and management. Paediatr Drugs. 2014;16(6): 447-56.

9. Adler A, Novelli V, Amin AS, Abiusi E, Care M, Nannenberg EA, et al. An international, multicentered, evidencebased reappraisal of genes reported to cause congenital long QT syndrome. Circulation. 2020;141:418-28.

10. Hodgkin AL, Huxley AF. A quantitative description of membrane current and its application to conduction and excitation in nerve. J Physiol. 1952; 117:500-44.

11. Hodgkin AL, Huxley AF. The components of membrane conductance in the giant axon of Loligo. J Physiol. 1952;116:473-96.

12. Hodgkin AL, Huxley AF, Katz B. Measurement of current-voltage relations in the membrane of the giant axon of Loligo. J Physiol. 1952;116:424-48.

13. Neher E, Sakmann B. Single-channel currents recorded from membrane of denervated frog muscle fibres. Nature. 1976;260(5554):799-802.

14. Hamill OP, Marty A, Neher E, Sakmann B, Sigworth FJ. Improved patchclamp techniques for high resolution current recording from cells and cellfree membrane patches. Pflugers Arch. 1981;391:85-100.

15. Mitcheson JS, Sanguinetti MC. Biophysical properties and molecular basis of cardiac rapid and slow delayed rectifier K channels. Cell Physiol Biochem. 1999;9:201-16

16. Sanguinetti MC, Jurkiewicz NK. Two components of cardiac delayed rectifier $K^{+}$current. J Gen Physiol. 1990;96:195-215.

17. Sanguinetti MC, Jurkiewicz NK. Lanthanum blocks a specific component of $I_{k}$ and screens membrane surface charge in cardiac cells. Am J Phys. 1990; 259:H1881-9.

18. Wang Q, Curran ME, Splawski I, Burn TC, Millholland JM, VanRaay TJ, et al. Positional cloning of a novel potassium channel gene: KVLQT1 mutations cause cardiac arrhythmias. Nat Genet. 1996;12:17-23.

19. Chouabe C, Drici MD, Romey G, Barhanin J, Lazdunski M. HERG and KvLQT!/ ISK, the cardiac $\mathrm{K}^{+}$channels involved in long QT syndromes are targets for calcium channel blockers. Mol Pharmacol. 1998:54(4):695-703.

20. Curran ME, Splawski I, Timothy KW, Vincent GM, Green ED, Keating MT. A molecular basis for cardiac arrhythmia: HERG mutations cause long QT syndrome. Cell. 1995;80:795-803.

21. Sanguinetti MC, Jiang C, Curran ME, Keating MT. A mechanistic link between an inherited and an acquired cardiac arrhythmia: HERG encodes the $I_{\text {kr }}$ potassium channel. Cell. 1995;81:299-307.

22. Trudeau MC, Warmke JW, Ganetzky B, Robertson GA. HERG, an inward rectifier in the voltage-gated potassium channel family. Science. 1995;269:92-5.

23. Sanguinetti MC, Curran ME, Spector PS, Keating MS. Spectrum of HERG K channel dysfunction in an inherited cardiac arrhythmia. Proc Natl Acad Sci U S A. 1996;93:2208-12.

24. Wang Q, Shen J, Splawski I, Atkinson D, Li Z, Robinson JL, et al. SCN5A mutations associated with an inherited cardiac arrhythmia, long QT syndrome. Cell. 1995;80:805-11.

25. Bennett PB, Yazawa K, Makita N, George AJ. Molecular mechanism for an inherited cardiac arrhythmia. Nature. 1995;376:683-5.

26. Wang Q, Shen J, Li Z, Timothy K, Vincent GM, Priori SG, et al. Cardiac sodium channel mutations in patients with long QT syndrome, an inherited cardiac arrhythmia. Hum Molec Gen. 1995;4:1603-7.

27. Chen QY, Kirsch GE, Zhang DM, Brugada R, Brugada J, Brugada P, et al. Genetic basis and molecular mechanism for idiopathic ventricular fibrillation. Nature. 1998;392:293-4.
28. Hancox JC, MCPate MJ, El Harchi A, Zhang YH. The hERG potassium channel and hERG screening for drug-induced torsades de pointes. Pharmacol Ther. 2008;119:118-32.

29. Gintant GA. Preclinical Torsades-de-pointes screens: advantages and limitations of surrogate and direct approaches in evaluating proarrhythmic risk. Pharmacol Ther. 2008;119(2):199-209.

30. Fermini B, Hancox JC, Bi-Gerges N, Bridgland-Taylor M, Chaudhary KW, Colatsky T, et al. A new perspective in the field of cardiac safety testing through the comprehensive in vitro Proarrhythmia assay paradigm. J Biomol Screen. 2016;21(1):1-11.

31. Smith JL, Anderson CL, Burgess DE, Elayi CS, January $C T$, Delisle BP. Molecular pathogenesis of long QT syndrome type 2. J Arrhythm. 2016;32(5):373-80.

32. Anderson CL, Delisle BP, Anson BD, Kilby JA, Will ML, Tester DJ, et al. Most LQT2 mutations reduce Kv11.1 (hERG) current by a class 2 (traffickingdeficient) mechanism. Circulation. 2006:113(3):365-73.

33. Anderson CL, Kuzmicki CE, Childs RR, Hintz CJ, Delisle BP, January CT. Largescale mutational analysis of Kv11.1 reveals molecular insights into type 2 long QT syndrome. Nat Commun. 2014;5:5535.

34. Vanoye CG, Desai RR, Fabre KL, Gallagher SL, Potet F, Dekeyser JM, et al. High-throughput functional evaluation of KCNQ1 decrypts variants of unknown significance. Circ Genom Precis Med. 2018;11(11):e002345.

35. Ng CA, Perry MD, Liang W, Smith NJ, Foo B, Shrier A, et al. High-throughput phenotyping of heteromeric human ether-a-go-go-related gene potassium channel variants can discriminate pathogenic from rare benign variants. Heart Rhythm. 2020;17(3):492-500.

36. Glazer AM, Wada Y, Li B, Muhammed A, Kalash OR, O'Neill MJ, et al. Highthroughput reclassification of SCN5A variants. bioRxiv. 2019:1-39. https://doi. org/10.1101/858175

37. Stenson PD, Mort M, Ball EV, Evans K, Hayden M, Heywood S, et al. The human gene mutation database: towards a comprehensive repository of inherited mutation data for medical research, genetic diagnosis and nextgeneration sequencing studies. Hum Genet. 2017;136(6):665-77.

38. Clemens DJ, Lentino AR, Kapplinger JD, Ye D, Zhou W, Tester DJ, et al. Using the genome aggregation database, computational pathogenicity prediction tools, and patch clamp heterologous expression studies to demote previously published long QT syndrome type 1 mutations from pathogenic to benign. Heart Rhythm. 2018;15(4):555-61.

39. Moss AJ, Zareba W, Kaufman ES, Gartman E, Peterson DR, Benhorin J, et al. Increased risk of arrhythmic events in long-QT syndrome with mutations in the pore region of the human ether-a-go-go-related gene potassium channel. Circulation. 2002;105(7):794-9.

40. Shimizu W, Moss AJ, Wilde AA, Towbin JA, Ackerman MJ, January CT, et al. Genotype-phenotype aspects of type 2 long QT syndrome. J Am Coll Cardiol. 2009;54(22):2052-62.

41. Kroncke BM, Glazer AM, Smith DK, Blume JD, Roden DM. SCN5A (Nav1.5) Variant Functional Perturbation and Clinical Presentation: Variants of a Certain Significance. Circ Genom Precis Med. 2018;11(5):e002095.

42. Viero C, Thomas NL, Euden J, Mason SA, George CH, Williams AJ. Techniques and methodologies to study the ryanodine receptor at the molecular, subcellular and cellular level. Adv Exp Med Biol. 2012;740:183-215.

43. Chai S, Wan X, Ramirez-Navarro A, Tesar PJ, Kaufman ES, Ficker E, et al. Physiological genomics identifies genetic modifiers of long QT syndrome type 2 severity. J Clin Invest. 2018;128(3):1043-56.

44. Nerbonne JM, Nichols CG, Schwarz TL, Escande D. Genetic manipulation of cardiac $\mathrm{K}^{+}$channel function in mice: what have we learned, and where do we go from here? Circ Res. 2001;89(11):944-56.

45. Anon. ICH S7B Note for guidance on the nonclinical evaluation of the potential for delayed ventricular repolarization (QT interval prolongation) by human pharmaceuticals. 2005. London, 25 May. Reference CHMP//CH/423/02.

46. Brunner M, Peng X, Liu GX, Ren XQ, Ziv O, Choi BR, et al. Mechanisms of cardiac arrhythmias and sudden death in transgenic rabbits with long QT syndrome. J Clin Invest. 2008;118(6):2246-59.

47. Odening KE, Bodi I, Franke G, Rieke R, de MA R, Perez-Feliz S, et al. Transgenic short-QT syndrome 1 rabbits mimic the human disease phenotype with QT/action potential duration shortening in the atria and ventricles and increased ventricular tachycardia/ventricular fibrillation inducibility. Eur Heart J. 2018;40(10):842-53.

48. Odening KE, Hyder O, Chaves L, Schofield L, Brunner M, Kirk M, et al. Pharmacogenomics of anesthetic drugs in transgenic LQT1 and LQT2 rabbits reveal genotype-specific differential effects on cardiac repolarization. Am J Physiol Heart Circ Physiol. 2008;295(6):H2264-72. 
49. Odening KE, Kirk M, Brunner M, Ziv O, Lorvidhaya P, Liu GX, et al. Electrophysiological studies of transgenic long QT type 1 and type 2 rabbits reveal genotype-specific differences in ventricular refractoriness and his conduction. Am J Physiol Heart Circ Physiol. 2010;299(3):H643-55.

50. Langheinrich U, Vacun G, Wagner T. Zebrafish embryos express an orthologue of HERG and are sensitive toward a range of QT-prolonging drugs inducing severe arrhythmia. Toxicol Appl Pharmacol. 2003;193(3):370-82.

51. Meder B, Scholz EP, Hassel D, Wolff C, Just S, Berger IM, et al. Reconstitution of defective protein trafficking rescues long-QT syndrome in zebrafish. Biochem Biophys Res Commun. 2011;408(2):218-24.

52. Liu CC, Li L, Lam YW, Siu CW, Cheng SH. Improvement of surface ECG recording in adult zebrafish reveals that the value of this model exceeds our expectation. Sci Rep. 2016;6:25073.

53. Jou CJ, Barnett SM, Bian JT, Weng HC, Sheng X, Tristani-Firouzi M. An in vivo cardiac assay to determine the functional consequences of putative long QT syndrome mutations. Circ Res. 2013;112(5):826-30.

54. Tanaka $Y$, Hayashi K, Fujino N, Konno $T$, Tada $H$, Nakanishi $C$, et al. Functional analysis of $\mathrm{KCNH} 2$ gene mutations of type 2 long QT syndrome in larval zebrafish using microscopy and electrocardiography. Heart Vessel. 2019;34(1):159-66.

55. Abramochkin DV, Hassinen M, Vornanen M. Transcripts of Kv7.1 and MinK channels and slow delayed rectifier $\mathrm{K}^{+}$current $\left(\mathrm{I}_{\mathrm{KS}}\right)$ are expressed in zebrafish (Danio rerio) heart. Pflugers Arch. 2018;470(12):1753-64.

56. Wu C, Sharma K, Laster K, Hersi M, Torres C, Lukas TJ, et al. Kenq1-5 (Kv7.1-5) potassium channel expression in the adult zebrafish. BMC Physiol. 2014;14:1.

57. Huttner IG, Trivedi G, Jacoby A, Mann SA, Vandenberg Jl, Fatkin D. A transgenic zebrafish model of a human cardiac sodium channel mutation exhibits bradycardia, conduction-system abnormalities and early death. J Mol Cell Cardiol. 2013;61:123-32.

58. Davis RP, Casini S, van den Berg CW, Hoekstra M, Remme CA, Dambrot C, et al. Cardiomyocytes derived from pluripotent stem cells recapitulate electrophysiological characteristics of an overlap syndrome of cardiac sodium channel disease. Circulation. 2012;125(25):3079-91.

59. Hoekstra M, Mummery CL, Wilde AA, Bezzina CR, Verkerk AO. Induced pluripotent stem cell derived cardiomyocytes as models for cardiac arrhythmias. Front Physiol. 2012;3:346.

60. Yoshinaga D, Baba S, Makiyama T, Shibata H, Hirata T, Akagi K, et al. Phenotype-based high-throughput classification of long QT syndrome subtypes using human induced pluripotent stem cells. Stem Cell Reports. 2019;13(2):394-404.

61. Bellin M, Greber B. Human iPS cell models of Jervell and Lange-Nielsen syndrome. Rare Dis. 2015;3(1):e1012978.

62. Garg P, Oikonomopoulos A, Chen H, Li Y, Lam CK, Sallam K, et al. Genome editing of induced pluripotent stem cells to decipher cardiac channelopathy variant. J Am Coll Cardiol. 2018;72(1):62-75.

63. Itzhaki I, Maizels L, Huber I, Zwi-Dantsis L, Caspi O, Winterstern A, et al. Modelling the long QT syndrome with induced pluripotent stem cells. Nature. 2011:471(7337):225-9.

64. Bellin M, Casini S, Davis RP, D'Aniello C, Haas J, Ward-van OD, et al. Isogenic human pluripotent stem cell pairs reveal the role of a $\mathrm{KCNH} 2$ mutation in long-QT syndrome. EMBO J. 2013;32(24):3161-75.

65. Matsa E, Rajamohan D, Dick E, Young L, Mellor I, Staniforth A, et al. Drug evaluation in cardiomyocytes derived from human induced pluripotent stem cells carrying a long QT syndrome type 2 mutation. Eur Heart J. 2011;32(8):952-62.

66. Mehta A, Sequiera GL, Ramachandra CJ, Sudibyo Y, Chung Y, Sheng J, et al. Re-trafficking of hERG reverses long QT syndrome 2 phenotype in human iPS-derived cardiomyocytes. Cardiovasc Res. 2014;102(3):497-506.

67. Mesquita FCP, Arantes PC, Kasai-Brunswick TH, Araujo DS, Gubert F, Monnerat G, et al. R534C mutation in hERG causes a trafficking defect in iPSC-derived cardiomyocytes from patients with type 2 long QT syndrome. Sci Rep. 2019;9(1):19203.

68. Sala L, Gnecchi M, Schwartz PJ. Long QT syndrome Modelling with Cardiomyocytes derived from human-induced pluripotent stem cells. Arrhythm Electrophysiol Rev. 2019;8(2):105-10.

69. den BL v, Grandela C, Mummery CL, Davis RP. Inherited cardiac diseases, pluripotent stem cells, and genome editing combined-the past, present, and future. Stem Cells. 2020;38(2):174-86.

70. Bett GC, Kaplan AD, Lis A, Cimato TR, Tzanakakis ES, Zhou Q, et al. Electronic "expression" of the inward rectifier in cardiocytes derived from humaninduced pluripotent stem cells. Heart Rhythm. 2013;10(12):1903-10.

71. Ben-Ari M, Naor S, Zeevi-Levin N, Schick R, Ben JR, Reiter I, et al. Developmental changes in electrophysiological characteristics of human- induced pluripotent stem cell-derived cardiomyocytes. Heart Rhythm. 2016; 13(12):2379-87.

72. Hill AP, Perry MD, Bi-Gerges N, Couderc JP, Fermini B, Hancox JC, et al. Computational cardiology and risk stratification for sudden cardiac death: one of the grand challenges for cardiology in the 21st century. J Physiol. 2016:594(23):6893-908.

73. ten Tusscher KH, Noble D, Noble PJ, Panfilov AV. A model for human ventricular tissue. Am J Physiology. 2004;286:H1573-89.

74. O'Hara T, Virag L, Varro A, Rudy Y. Simulation of the undiseased human cardiac ventricular action potential: model formulation and experimental validation. PLoS Comput Biol. 2011;7(5):e1002061.

75. Fink M, Noble D, Virag L, Varro A, Giles WR. Contributions of HERG K ${ }^{+}$ current to repolarization of the human ventricular action potential. Prog Biophys Mol Biol. 2008;96(1-3):357-76.

76. Grandi E, Pasqualini FS, Bers DM. A novel computational model of the human ventricular action potential and Ca transient. J Mol Cell Cardiol. 2010;48(1):112-21.

77. Smith JL, Tester DJ, Hall AR, Burgess DE, Hsu CC, Elayi SC, et al. Functional Invalidation of putative sudden infant death syndrome-associated variants in the KCNH2-Encoded Kv11.1 Channel. Circ Arrhythm Electrophysiol. 2018; 11(5):e005859.

78. Zhou Z, Gong Q, Ye B, Fan Z, Makielski JC, Robertson GA, et al. Properties of HERG channels stably expressed in HEK 293 cells studied at physiological temperature. Biophys J. 1998;74:230-41.

79. Hancox JC, Levi AJ, Witchel HJ. Time course and voltage dependence of expressed HERG current compared with native 'rapid' delayed rectifier K current during the cardiac ventricular action potential. Pflugers Archiv - Eur J Physiol. 1998;436:843-53.

80. Lu Y, MahautSmith MP, Varghese A, Huang CLH, Kemp PR, Vandenberg Jl. Effects of premature stimulation on HERG channels. J Physiol. 2001;537(3): 843-51.

81. Noble D, Varghese A, Kohl P, Noble P. Improved guinea-pig ventricular cell model incorporating a diadic space, Ikr and Iks, and length- and tensiondependent processes. Can J Cardiol. 1998;14(1):123-34.

82. Al Moubarak E, Zhang Y, Dempsey CE, Zhang H, Harmer SC, Hancox JC. Serine mutation of a conserved threonine in the hERG $\mathrm{K}^{+}$channel S6-pore region leads to loss-of-function through trafficking impairment. Biochem Biophys Res Commun. 2020;526(4):1085-91.

83. Milnes JT, Witchel HJ, Leaney JL, Leishman DJ, Hancox JC. Investigating dynamic protocol-dependence of hERG potassium channel inhibition at 37 degrees C: Cisapride versus dofetilide. J Pharmacol Toxicol Methods. 2010; 61(2):178-91.

84. Brnich SE, bou Tayoun AN, Couch FJ, Cutting GR, Greenblatt MS, Heinen CD, et al. Recommendations for application of the functional evidence PS3/BS3 criterion using the ACMG/AMP sequence variant interpretation framework. Genome Med. 2019;12(1):3.

85. Nykamp K, Anderson M, Powers M, Garcia J, Herrera B, Ho YY, et al. Sherloc: a comprehensive refinement of the ACMG-AMP variant classification criteria. Genet Med. 2017;19(10):1105-17.

86. Dong J, Williams N, Cerrone M, Borck C, Wang D, Zhou B, et al. Molecular autopsy: using the discovery of a novel de novo pathogenic variant in the KCNH2 gene to inform healthcare of surviving family. Heliyon. 2018;4(12):e01015.

87. Crotti L, Lundquist AL, Insolia R, Pedrazzini M, Ferrandi C, De Ferrari GM, et al. KCNH2-K897T is a genetic modifier of latent congenital long-QT syndrome. Circulation. 2005;112(9):1251-8.

88. Roberts JD, Asaki SY, Mazzanti A, Bos JM, Tuleta I, Muir AR, et al. An international multi-center evaluation of type 5 long QT syndrome: a low penetrant primary arrhythmic condition. Circulation. 2020;141(6):429-39.

89. Harmer SC, Tinker A. The role of abnormal trafficking of KCNE1 in long QT syndrome 5. Biochem Soc Trans. 2007;35(Pt 5):1074-6.

90. Harmer SC, Wilson AJ, Aldridge R, Tinker A. Mechanisms of disease pathogenesis in long QT syndrome type 5. Am J Physiol Cell Physiol. 2010; 298(2):C263-73.

91. McDonald TV, Yu Z, Ming Z, Palma E, Meyers MB, Wang KW, et al. A minK-HERG

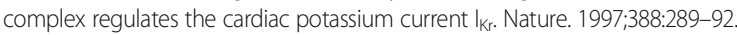

92. Nishio $Y$, Makiyama T, Itoh H, Sakaguchi T, Ohno S, Gong YZ, et al. D85N, a KCNE1 polymorphism, is a disease-causing gene variant in long QT syndrome. J Am Coll Cardiol. 2009;54(9):812-9.

93. Ohno S, Zankov DP, Yoshida H, Tsuji K, Makiyama T, Itoh H, et al. N- and Cterminal KCNE1 mutations cause distinct phenotypes of long QT syndrome. Heart Rhythm. 2007;4(3):332-40. 
94. Du C, El Harchi A, Zhang H, Hancox JC. Modification by KCNE1 variants of the $h E R G$ potassium channel response to premature stimulation and to pharmacological inhibition. Physiol Rep. 2013;1(6):e00175.

95. McCrossan ZA, Abbott GW. The MinK-related peptides. Neuropharmacology. 2004;47(6):787-821.

96. Crump SM, Abbott GW. Arrhythmogenic KCNE gene variants: current knowledge and future challenges. Front Genet. 2014;5:3.

97. Kelly MA, Caleshu C, Morales A, Buchan J, Wolf Z, Harrison SM, et al. Adaptation and validation of the ACMG/AMP variant classification framework for MYH7-associated inherited cardiomyopathies: recommendations by ClinGen's inherited cardiomyopathy expert panel. Genet Med. 2018;20(3):351-9.

\section{Publisher's Note}

Springer Nature remains neutral with regard to jurisdictional claims in published maps and institutional affiliations.

Ready to submit your research? Choose BMC and benefit from:

- fast, convenient online submission

- thorough peer review by experienced researchers in your field

- rapid publication on acceptance

- support for research data, including large and complex data types

- gold Open Access which fosters wider collaboration and increased citations

- maximum visibility for your research: over $100 \mathrm{M}$ website views per year

At BMC, research is always in progress.

Learn more biomedcentral.com/submissions 